(C) 2003 IEEE. Personal use of this material is permitted. Permission from IEEE must be obtained for all other uses, in any current or future media, including reprinting/republishing this material for advertising or promotional purposes, creating new collective works, for resale or redistribution to servers or lists, or reuse of any copyrighted component of this work in other works. 


\title{
Core Losses in Claw Pole Permanent Magnet Machines with Soft Magnetic Composite Stators
}

\author{
YouGuang GUO, Jian Guo ZHU, Senior Member, IEEE, Jin Jiang ZHONG, and Wei WU
}

\begin{abstract}
This paper presents the core loss calculation in SMC sample and a claw pole permanent magnet machine with SMC stator. By using finite element analysis of magnetic field, the total core loss is computed by separating the hysteresis (alternating and rotational, both purely circular and elliptical), eddy current, and anomalous losses in each element, when the rotor rotates. The coefficients for each loss component are determined by a loss separation procedure and the experimental data obtained by a single sheet two-dimensional core loss testing system.
\end{abstract}

Index Terms--Soft magnetic composite materials, core losses, claw pole machines, finite element analysis.

\section{INTRODUCTION}

S oft magnetic composite (SMC) materials produced by a $S_{\text {powder metallurgy technique are very useful for }}$ construction of three-dimensional (3D) flux electromagnetic devices due to their isotropic magnetic and thermal properties, low eddy current loss, and nearly net-shape fabrication process with good tolerance and surface finish (no need of further machining) [1]. Although the eddy current loss component is very low, the SMC materials have a significantly greater total core loss than electrical steels at low frequencies. Therefore, accurate core loss calculation and its reduction are of major concern to electrical machine designers.

The local flux density patterns within a 3D flux electrical machine are quite complex [2]. The flux density locus at one position can be alternating (1D) with or without harmonics, two-dimensional (2D) or even 3D rotating with purely circular or elliptical patterns. Experiments on samples have revealed significant differences between the core losses caused by an alternating and a rotating magnetic field [3,4]. Core losses should be considered properly in the optimum design of electrical machines.

This paper firstly reports the measurement and calculation models of power losses in a square SMC sample under different patterns of flux density. The models and coefficients deduced from the experimental data are then used together with the magnetic field finite element analysis to calculate the core losses in a claw pole permanent magnet (PM) machine

Y.G. Guo, J.G. Zhu, and J.J. Zhong are with the Faculty of Engineering, University of Technology, Sydney, NSW 2007, Australia (e-mail: youguang.guo-1@.uts.edu.au, jianguo.zhu@uts.edu.au,).

W. Wu is with CSIRO Telecommunications and Industrial Physics, NSW 2070, Australia (e-mail: wei.wu@csiro.au). with a SMC stator. The theoretical result is compared with experiments on a prototype machine.

\section{CORE LOSS MEASUREMENT IN SMC SAMPLE}

Alternating and rotational core losses in a SMC sample are obtained via the measurement of magnetic field intensity $\boldsymbol{H}$ and flux density $\boldsymbol{B}$ with a single sheet two-dimensional core loss tester [3]. Fig. 1 shows the testing system schematically. The DSP system is a PC based digital signal processing system for both data acquisition and function generation. The flux density waveforms on the $\mathrm{X}$ - and $\mathrm{Y}$-axes are controlled by negative feedback loops to track the signals generated by the DSP system.

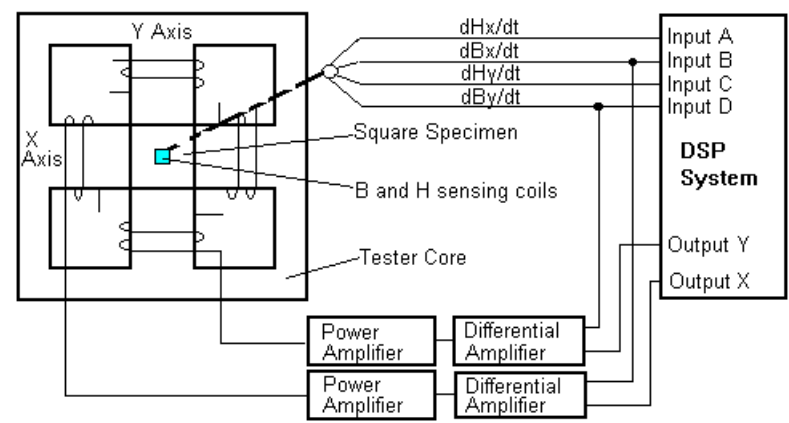

Fig. 1. Schematic diagram of the square specimen testing system

By Poynting's theorem, the total core loss $P_{t}$ in the sample can be calculated by

$$
P_{t}=\frac{1}{T \rho_{m}} \int_{0}^{T} \mathbf{H} \bullet \frac{d \mathbf{B}}{d t} d t
$$

where $T$ is the time period of magnetization and $\rho_{m}$ the mass density of sample.

Figs. 2(a) and (b) illustrate the measured alternating and purely rotational core losses of a SMC block sample with dimensions $50 \mathrm{~mm} \times 50 \mathrm{~mm} \times 1.27 \mathrm{~mm}$. The sample material used is SOMALOY ${ }^{\mathrm{TM}} 500$, a SMC product developed by Höganäs $\mathrm{AB}$, Sweden. It uses a highly pure iron particle with a surface coating to ensure low eddy current loss. The coated iron powder is bonded and then pressed into a die to form the required net shape and finally heat-treated to anneal and cure the bond. This material is especially suitable for construction of electromagnetic devices with 3D fluxes and complex structures such as claw pole and transverse flux machines. 


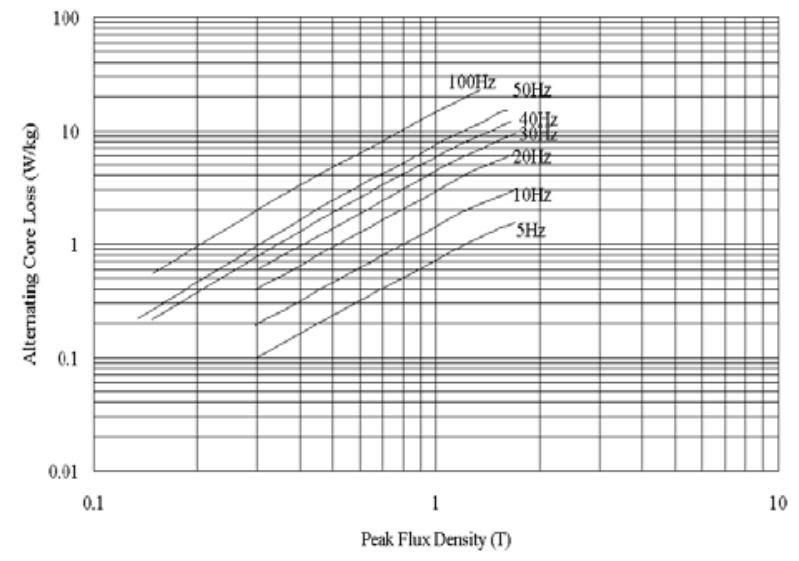

(a)

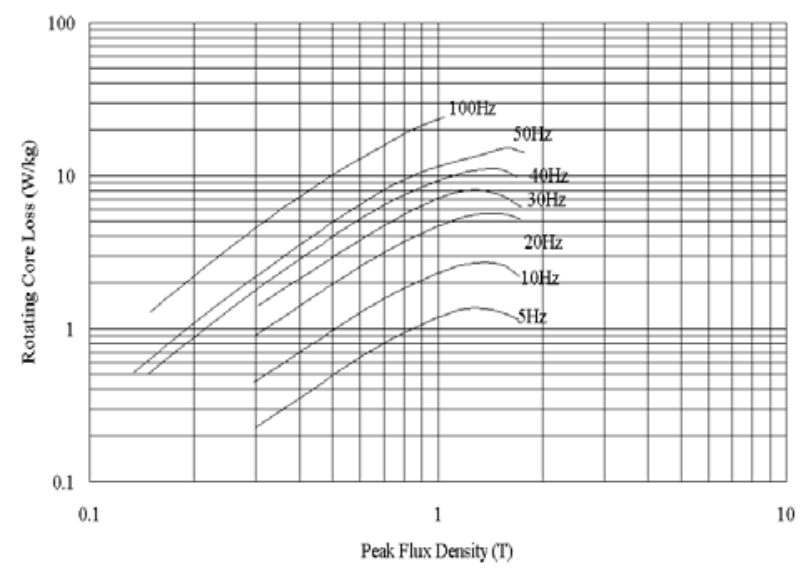

(b)

Fig. 2. Core losses of SMC sample with (a) alternating \& (b) circular rotating fluxes at different frequencies

\section{MODELING OF CORE LOSSES IN SMC}

For alternating (pulsating) core loss modeling, a standard practice is to separate the loss into the following three components: hysteresis, eddy current and anomalous losses, i.e. $P_{a}=C_{h a} f B^{h}+C_{e a}(f B)^{2}+C_{a a}(f B)^{1.5}$, where $B$ is the peak value of flux density, $f$ the frequency, and $C_{h a}, h, C_{e a}$, and $C_{a a}$ are the loss coefficients.

By fitting the model to the experimental results in Fig. 2(a), these constants are deduced as $C_{h a}=0.1402, h=1.548$, $C_{e a}=1.233 \times 10^{-5}$, and $C_{a a}=3.645 \times 10^{-4}$.

Similarly, the specific circular core loss can also be separated into three parts as $P_{a}=P_{h r}+C_{e r}(f B)^{2}+C_{a r}(f B)^{1.5}$, where $P_{h r}$ is the rotational hysteresis loss, and $C_{e r}$ and $C_{a r}$ are the coefficients for the rotational eddy current and anomalous losses. The rotational hysteresis loss behaves very differently from its alternating counterpart. A rotational field causes nearly twice the loss, compared to the loss produced by an alternating field with the same peak value at a mid-range flux density. However, at saturation the loss caused by a rotating field falls markedly to the levels well below that caused by an alternating field [4]. To model the rotational hysteresis loss, a formulation was proposed in [4]. It is postulated that the specific rotational hysteresis loss per cycle can be expressed in terms of four parameters, $a_{1}, a_{2}, a_{3}$, and $B_{s}$, by
$\frac{P_{h r}}{f}=a_{1}\left[\frac{1 / s}{\left(a_{2}+1 / s\right)^{2}+a_{3}^{2}}-\frac{1 /(2-s)}{\left[a_{2}+1 /(2-s)\right]^{2}+a_{3}^{2}}\right]$

where

$$
s=1-\frac{B}{B_{s}} \sqrt{1-\frac{1}{a_{2}^{2}+a_{3}^{2}}}
$$

By fitting the above formulations to the rotational loss curves in Fig. 2(b), the coefficients for the tested SMC sample are $C_{e r}=2.303 \times 10^{-4}, C_{a r}=0, a_{1}=6.814, a_{2}=1.054, a_{3}=1.445$, and $B_{s}=2.134 \mathrm{~T}$.

Generally, the flux density loci in 3D flux machines are elliptical, including alternating $\left(R_{B}=0\right)$ and circular $\left(R_{B}=1\right)$, where $R_{B}=B_{\text {min }} / B_{\text {maj }}$ is the axis ratio of $\boldsymbol{B}, B_{\text {maj }}$ and $B_{\text {min }}$ are the major and minor axes of the elliptical $\boldsymbol{B}$ locus. The elliptical core loss can be predicted from the alternating and purely circular formulations as [4]: $P_{t}=R_{B} P_{r}+\left(1-R_{B}\right)^{2} P_{a}$, where $P_{r}$ and $P_{a}$ are the core losses with a circular $\boldsymbol{B}$ and an alternating $\boldsymbol{B}$ with $B_{m a j}=B_{p}$, respectively, and $B_{p}$ the peak value of the alternating $\boldsymbol{B}$.

\section{CORE LOSSES IN ELECTRICAL MACHINES}

\section{A. Magnetic Field Analysis and Core Loss Calculation}

To illustrate the method for core loss analysis, an SMC claw pole PM machine has been chosen as an example. Fig. 3 shows an outline of the prototype [2]. It is a single-phase twenty-pole surface mounted NdFeB PM machine. The stator core is made of SOMALOY'M 500 and the rotor of mild steel.

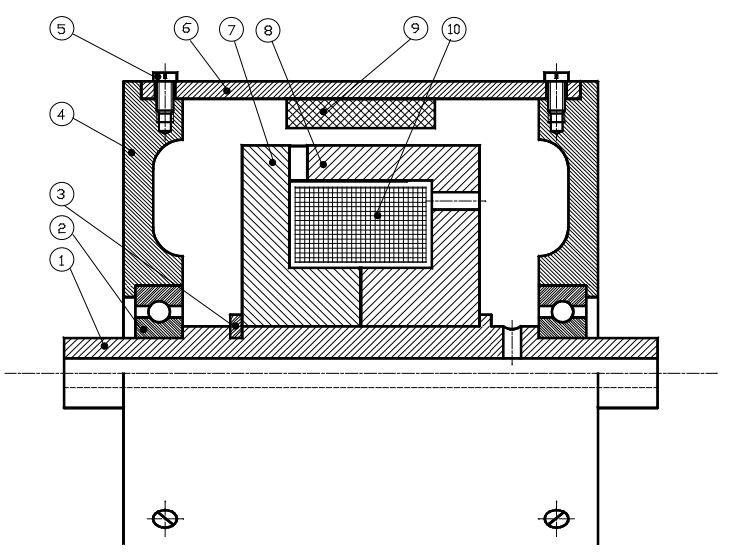

Fig. 3. Claw pole machine prototype assembly, where (1) is the shaft, (2) are the bearings, (3) is the thrust washer, (4) are the aluminum end plates, (5) the screws, (6) is the mild steel rotor yoke, (7) and (8) are the SOMALOY ${ }^{\mathrm{TM}} 500$ claw poles and the stator core, (9) $20 \mathrm{NdFeB}$ permanent magnets, and (10) is the stator winding

The complex shape of the claw pole machine leads to a truly 3D magnetic flux, and 3D finite element analysis is necessary for accurate determination of the parameters and performance of the machine. Because of the symmetry, only one pole pitch region is needed for field solution and the full flux density locus for any element can be obtained as the rotor rotates 
through only half a pole pitch. At the two radial boundary planes, the magnetic scalar potential used to solve the magnetic field distribution obeys the so-called half-periodical boundary conditions:

$$
\varphi_{m}(r, \Delta \theta / 2, z)=-\varphi_{m}(r,-\Delta \theta / 2,-z)
$$

where $\Delta \theta=18^{\circ}$ is the angle of one pole pitch. The original point of the cylindrical coordinate is located at the center of the motor.

Figs. 4(a), (b), and (c) show the calculated 3D flux density loci in three typical elements, the claw pole, stator yoke and side plate. It is shown that the flux density in the claw pole and side plates is truly three-dimensional and generally elliptically rotational. Fig. 5 shows the core loss calculated at different frequencies or rotor speeds.

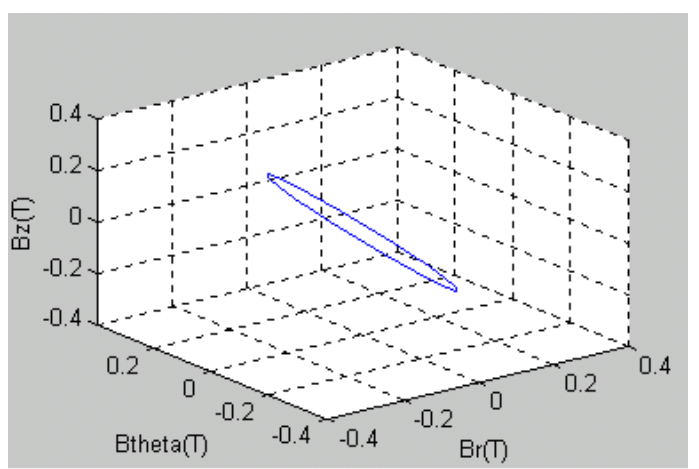

(a)

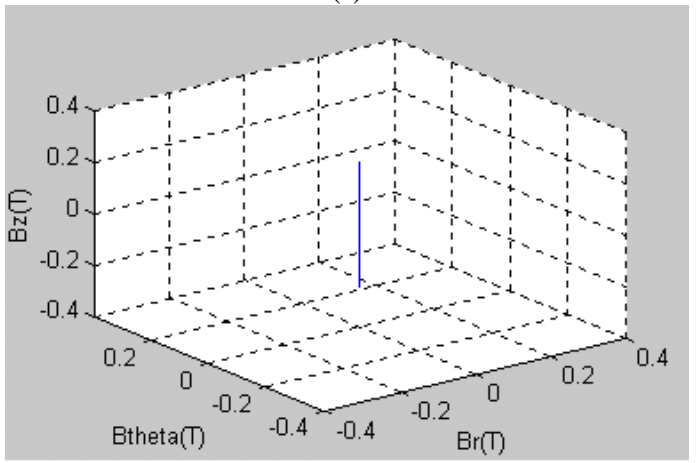

(b)

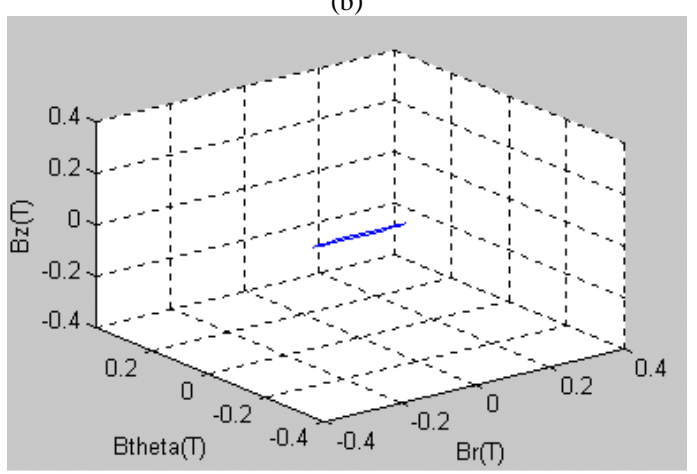

(c)

Fig. 4. Flux density loci at typical elements of (a) the claw poles, (b) stator yoke, and (c) side plates. Br, Btheta, and $\mathrm{Bz}$ are the radial, circumferential, and axial components of flux density, respectively.

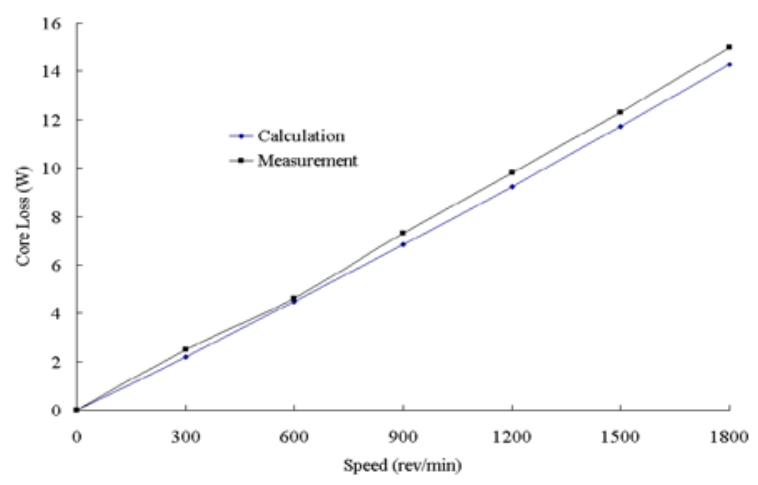

Fig. 5. Core loss calculation and measurement of SMC machine

\section{B. Core Loss Measurement}

To measure the core loss of the claw pole PM machine, a calibrated dc motor is used as a prime mover. The power fed into the dc motor is measured under two conditions: standalone and when coupled to the claw pole PM motor. The difference of the measured input power gives the total of the core loss and mechanical loss of the tested machine.

The mechanical loss of the claw pole PM machine is measured by replacing the SMC stator with a wooden tube (to imitate the windage) and then repeating the previous test procedure. The core loss is obtained by subtracting the mechanical loss from the total of the core loss and mechanical loss. The measured core losses at different speeds are also shown in Fig. 5. The calculation core loss is about 6\% lower than the measured. This may be attributed to the core loss in the stator shaft and other iron parts not included in the calculation, which is also relevant to the flux density variation frequency or the rotor speed.

\section{CONCLUSION}

The comparison between the calculated and measured core losses in the SMC claw pole machine shows that the proposed core loss models and the calculation method are practical.

\section{ACKNOWLEDGMENT}

The authors wish to thank Höganäs $A B$, Sweden for providing the preforms of SOMALOY ${ }^{\mathrm{TM}} 500$. Thanks also go to Dr. P.A. Watterson at University of Technology, Sydney for his advice on the measurement of the prototype core losses.

\section{REFERENCES}

[1] A.G. Jack, "Experience with the Use of Soft Magnetic Composites in Electrical Machines," in Proceeding of International Conference on Electrical Machines, Istanbul, Turkey, 1998, pp. 1441-1448.

[2] Y.G. Guo and J.G. Zhu, "Magnetic Field Calculation of Claw Pole Permanent Magnet Machines Using Magnetic Network Method," Journal of Electrical \& Electronics Engineering, Australia, Vol.22, No.1, 2002, pp. 69-75.

[3] J.G. Zhu and V.S. Ramsden, "Two-dimensional Measurement of Magnetic Field and Core Loss Using a Square Specimen Tester," IEEE Transactions on Magnetics, Vol.29, No.6, November 1993, pp. 29952997.

[4] J.G. Zhu and V.S. Ramsden, "Improved Formulations for Rotational Core Losses in Rotating Electrical Machines," IEEE Transactions on Magnetics, Vol.34, No.4, July 1998, pp. 2234-2242. 